\title{
Perspectives
}

\section{Semen analysis: a new manual and its application to the understanding of semen and its pathology}

Anne M. Jequier

PIVET Medical Centre, Leederville, Western Australia 6007, Australia

\begin{abstract}
This article reviews the latest edition of the World Health Organization's manual on semen analysis, a comprehensive instructional guide. The methodology used in the assessment of the usual variables in semen analysis is described, as are many of the less common, but very valuable, sperm function tests. Seminal fluid preparation techniques for procedures such as in vitro fertilization and intrauterine insemination are also outlined in the manual. In addition, it details many useful techniques for the assessment of seminal fluid. It will be a very useful manual for any laboratory that carries out analyses of seminal fluid.
\end{abstract}

Asian Journal of Andrology (2010) 12: 11-13. doi: 10.1038/aja.2009.12.

Keywords: laboratory practice, male infertility, semen analysis

\section{Introduction}

For many years now the World Health Organization (WHO) manuals have served as a primary resource for seminal fluid analysis procedures. However, these manuals have not been comprehensive, and they have focused mostly on the evaluation of male infertility. In the forthcoming fifth edition, the scope has been considerably expanded, making semen analysis much easier for the average medical laboratory scientist to understand.

As a result, this is by far the most comprehensive edition of the manual. For example, it includes information about abnormalities that may occur in the interactions between spermatozoa taken from an infertile male and cervical mucus as well as oocytes. The text is well referenced and provides relevant background. I consider this one the

Correspondence to: Dr Anne M. Jequier, PIVET Medical Centre, 166 Cambridge Street, Leederville, Western Australia 6007, Australia. Fax:+61-8-9382-4576_E-mail: ajequier@bigpond.net.au Received: 14 January 2009 Revised: 15 January 2009

Accepted: 20 January 2009 best instruction manuals on semen analysis ever published. The dedication of the book to the late Professor Geoffrey Waites is very apt. The new edition has been divided into four parts, each of which is reviewed below. Standard tests as well as less frequently used methods are detailed, each with a description of its limitations. Methods of sperm preparation for both intrauterine insemination and in vitro fertilization (IVF)/intra cytoplasmic sperm injection (ICSI) are now included. Quality control methods are carefully explained, with the help of statistical examples that are easy to understand even by a clinician.

\section{Part 1: Semen analysis}

This section begins with an overview of the process of semen analysis and points out that fertility or sperm production cannot be assessed on the basis of a single sample of semen. However, it fails to acknowledge that the cause of the changes in an abnormal semen analysis such as the impairment of sperm function or a reduction in sperm numbers cannot be determined from the examination of a semen sample [1] and therefore medical laboratory scientists should refrain from giving advice on treatment. 


\subsection{Collection of the semen sample}

In the section relating to the collection of a semen sample, the authors state that the sample should be collected in a room near the laboratory. However, it is common for patients to report that they feel much more comfortable producing the sample at home. Provided that the sample can be brought into the lab with $2-3 \mathrm{~h}$ and can be maintained at a temperature of at least $20^{\circ} \mathrm{C}$ before analysis, home collection is, in my view, permissible.

\subsection{Evaluation of the physical properties of semen}

This section examines the assessment of the physical properties of semen, such as liquefaction, viscosity, semen volume and seminal $\mathrm{pH}$. It also describes tests to evaluate the severity of abnormalities of these properties. In addition, the authors stress the need for careful mixing of seminal fluid before its examination to avoid the common problem of generation of artefacts due to poor mixing. It is frequently forgotten that mixing of the components of seminal fluid occurs only after ejaculation, and in the collection of a semen sample, that can only occur in the semen pot.

Agglutination of spermatozoa in an ejaculate is not uncommon, and the handling of this problem and its reporting is well described and illustrated.

\subsection{Determination of sperm motility}

The next part of this section involves the assessment of sperm motility. It recommends the use of a chamber of at least 20 microns deep for this type of analysis, as shallower chambers may impede sperm movement. This section describes a simple and easy technique for detecting motility without introducing errors.

\subsection{Determination of sperm vitality}

This section describes the use of the eosin-nigrosin test to determine sperm vitality, an aspect of semen analysis that is important but often omitted by many laboratories. This is accompanied by very nice pictures of spermatozoa, both positively and negatively stained. Another vitality test that is included in this edition is the hypoosmotic swelling (HOS) test. This procedure is becoming increasingly useful, not only in detecting the vitality of the spermatozoa in semen analysis but also in determining the selection of spermatozoa for ICSI.

\subsection{Counting spermatozoa in a semen sample}

Counting spermatozoa is often considered the most important part of semen analysis. However, it is in fact only one part, and all the facets of semen quality must be examined if the analysis is to be of any substantive value to the clinician. The manual describes several types of chambers as well as each of the grids. As many counting chambers are now available and they are generally expen- sive, the section on their care is an important and welcome addition to the manual. The very good description of the use of these chambers and the methods for calculating sperm count is valuable.

The diagnosis of azoospermia is now very important, as even a few dozen spermatozoa can be used in an IVF/ ICSI cycle (thus obviating the need for surgical sperm search). Today, too few laboratories take enough care in the diagnosis of azoospermia, and centrifugation is rarely carried out.

\subsection{Counting non-sperm cells}

The identification of white blood cells in a semen sample is important for the clinician. As it is often impossible to differentiate these cells from germinal cells, the manual's explanation of the counting of these cells is valuable.

\subsection{Assessment of sperm morphology}

This aspect of semen analysis is usually considered the most difficult one, and the manual properly describes it in greater detail. The concept of normal spermatozoa is discussed, and the staining of slides is described in some detail. The preferred stains are named, and the make-up of these stains is also included. Also included in this section is a methodology for the use of the peroxidase stain, which positively identifies white blood cells.

Unlike many of the earlier editions of the manual, there is now a large collection of photomicrographs depicting many of the morphological abnormalities of spermatozoa, germinal cells and white blood cells, as well as a group of morphologically diverse spermatozoa that are considered to be 'normal'. This inclusion will be of great value to many newcomers in the field of semen analysis, and the editors of the manual are to be congratulated on their careful selection of these illustrations.

\subsection{Testing for the presence of anti-sperm antibodies}

This area also makes up an important part of a semen analysis, and standard tests such as the 'mixed antiglobulin reaction (MAR)' test and the immuno-bead test are described in detail. The use of indirect tests is also carefully described, as this is often very confusing to many new laboratory scientists engaged in semen analysis.

\subsection{Optional procedures}

This section includes what are described as 'optional' procedures, including the calculation of many indices, mostly in the area of sperm morphology. However, many of these tests are not carried out by routine laboratories largely owing to a lack of expertise in this field as well as their expense. In this section, the assessment of the post-coital test can also be found; surprisingly, even the 'donated' mucus is mentioned. 
Biochemical assays including the measurement of fructose, zinc and epididymal glucosidase are outlined. These are valuable, as many laboratories no longer have the time or money to carry out these clinically important tests. There is also a substantial section on the detection and measurement of reactive oxygen species, an area that is clearly gaining importance in the understanding of semen pathology. Attention is also given to execution and application of sperm-oocyte interaction as well as the use of an assessment to measure the acrosome reaction. These procedures are illustrated by high-quality photomicrographs.

Overall, every important test used in semen analysis is covered in Part 1, and the clarity and simplicity of the presentation is excellent.

\section{Part 2: Sperm preparation}

The separation of spermatozoa from seminal plasma is now an important aspect of the work of an andrology laboratory. Such an exercise may be needed to prepare spermatozoa for tests of functioning or for the use of such spermatozoa in a treatment regime (for example, intrauterine insemination). All the commonly used methods are simply described and included in this part of the manual, as are the means of handling HIV-infected semen samples. The preparation of testicular and epididymal spermatozoa are also described in this section, together with the methodology needed to collect spermatozoa from men with retrograde ejaculation. Methods of cryopreservation are also described, although a slightly extended section on this procedure, particularly including the physiology of freezing, may have been beneficial for beginners.

\section{Part 3: Quality assurance}

This section describes the problem of quality assurance in relation to semen samples. Although this may not be of great importance to the clinical field, it is very important in many aspects of research. There is an excellent section on laboratory factors that influence quality assurance. The use of statistics in this context is clearly described and simple to understand. It must always be remembered that the execution of a semen analysis is a skilled procedure and that proper training in this exercise is very important. It is clear, however, that training helps to prevent spurious results and poor quality control.

\section{Appendix}

The appendix contains much useful information relating to equipment, stock solutions, record keeping and general risk management.

In addition, it includes a section about reference values in relation to semen variables. Although these reference ranges are useful for epidemiological studies relating to men's health, it is dangerous to assume that they indicate either fertility or infertility except when they are at the extremes of the range. As Eliasson [2] pointed out nearly 40 years ago, conception relates to probabilities rather than reference ranges. Put another way, conception is related to probability not to the certainty that reference ranges would seem to indicate. It must also be remembered that controlling both male as well as female fertility is female fecundity-and this cannot be assessed from a semen analysis.

It should also be pointed out again that semen analysis alone is a poor discriminator of fertility and cannot, except on relatively rare occasions, provide a diagnosis. That is, an abnormal semen analysis is not a diagnosis; it is just a physical sign. Thus, comments on the treatment of patients with an abnormal sperm count, even that below the fifth percentile, are misleading, given that a diagnosis cannot definitively be given in the laboratory.

\section{Conclusions}

Overall, this edition of the WHO manual is very much improved relative to the earlier edition. It should be the standard reference manual for all laboratories that carry out semen analysis and similar procedures. The editors should be congratulated on producing such an improved version.

\section{References}

1 Jequier AM. Is quality assurance in semen analysis still really necessary? A clinician's viewpoint. Hum Reprod 2005; 20: 2039-42.

2 Eliasson R. Standards for the investigation of human semen. Andrologia 1971; 3: 49-64. 